\title{
Castellanización y afrancesamiento de los nombres de los municipios catalanes
}

\section{Interference of Spanish and French in the name of the Catalan municipalities}

\author{
Jesús Burgueño
}

\begin{abstract}
RESUMEN
Se analiza la evolución de los nombres de los municipios catalanes en el marco del proceso de centralización política contemporánea. Se compara la grafía catalana anterior a la Nueva Planta (1715) con la forma escrita oficial a mediados del s. XIx, para comprobar el grado de castellanización experimentado. La misma metodología se aplica en la Cataluña francesa. En total se analizan 1.304 topónimos. Se llega a la conclusión que en la Cataluña española hubo una mayoritaria continuidad de la grafía (76\%), frente a un escaso $33 \%$ en la parte anexionada a Francia. Se distinguen 4 grados de alteración de los topónimos; la distorsión máxima alcanza sólo un 3,3\% de los casos del lado español frente al 51,2\% del francés. La abundante cartografía francesa referida a Cataluña muestra la misma proliferación de topónimos deformados.
\end{abstract}

Palabras Clave: toponimia; cartografía histórica; castellanización; afrancesamiento; Cataluña.

\section{Abstract}

The article analyses the evolution of the names of the Catalan municipalities as a part of the process of contemporary political centralization of Spain. To check the degree of interference from the Spanish Language, Catalan writing before the Nueva Planta (new system of government of Catalonia, in 1715) is compared with the official written form in the middle of the 19th century. The same methodology is applied to the French Catalo-

1 Universitat de Lleida. burgue@geosoc.udl.cat ORCID iD: http://orcid.org/0000-0001$6644-7313$

Copyright: @ 2017 CSIC. Este es un artículo de acceso abierto distribuido bajo los términos de la licencia de uso y distribución Creative Commons Attribution (CC-by) España 3.0. 
nia. Altogether we analyzed 1,304 place names. It can be concluded that in Catalonia (Spain) there was a major continuity of the spelling (76\%), compared to just a $33 \%$ in the territories joined to France. There are 4 degrees of alteration of place-names; the maximum distortion reaches only $3.3 \%$ of the cases by the Spanish side, compared to $51.2 \%$ in France. The abundant French cartography referred to Catalonia shows the same proliferation of deformed toponyms.

KEY WORDS: place names; historical cartography; Catalan; language interference.

Cómo citar este artículo/Citation: Burgueño, Jesús (2017): "Castellanización y afrancesamiento de los nombres de los municipios catalanes", Estudios Geográficos, LXXVIII/283, pp. 633-656

\section{INTRODUCCIÓN}

La toponimia refleja las tensiones interlingüísticas y vehicula la preminencia de la lengua hegemónica en cada situación histórica. En Cataluña, hasta la abolición de las instituciones y leyes propias (Constitucions) como consecuencia de la victoria borbónica sobre el bando austriacista en la guerra de Sucesión, la única toponimia empleada siempre fue genuinamente catalana. A partir de 1714 la influencia castellana - hasta entonces simplemente fruto de la proximidad y vitalidad de una lengua prestigiada y en plena expansión-adoptó visos de imposición en la mayoría de los usos formales. Es particularmente expresiva la presencia, en la Instrucción secreta de algunas cosas que deben tener presentes los corregidores del Principado de Cathaluña para el exercicio de sus empleos, de 1727, de una obligación explícita: «Pondrá el mayor cuidado en introducir la lengua castellana, a cuyo fin dará las providencias más templadas y dissimuladas, para que se consiga el efecto sin que se note el cuydado.» (Gay Escoda, 1997: 919).

Pero, ¿hasta qué punto y de qué manera se castellanizó la toponimia mayor de más trascendencia política: la denominación de los municipios? ¿Fue una castellanización radical y generalizada o, por el contrario, fue un mero barniz? Y en la vecina Francia, que obtuvo una porción de Cataluña con el Tratado de los Pirineos, ¿se produjo una situación homóloga a la española? Esta investigación pretende dar respuesta a estos interrogantes.

Para ello es preciso una aclaración metodológica previa: ¿qué debemos entender por "grafía catalana», cuando nos referimos a los siglos XVIII o XIX? Está claro que no puede tratarse de la normativa lingüística del Institut d'Estudis Catalans (IEC), hoy vigente, porque a menudo presenta soluciones 
que el catalán de época moderna no habría reconocido como propias: el uso del apóstrofe, el cambio del artículo masculino lo por el, la acentuación, etc. Si queremos valorar si, a partir de 1720, un determinado topónimo se castellanizó (o simplemente se deformó) debemos tomar como referencia las formas escritas inequívocamente catalanas inmediatamente anteriores. Al proceder de esta manera obtendremos una visión mucho más equilibrada de la cuestión, evitando magnificar la mutación experimentada por la tradición autóctona.

\section{La castellanización a partir de la Nueva Planta}

La lengua castellana prácticamente no había generado formas toponímicas propias y genuinas referidas a localidades catalanas antes de la asimilación jurídica de la Corona de Aragón, pero sí contaba con tres topónimos adaptados, tomados de formas arcaicas surgidas en el propio territorio: Gerona, Lérida y Urgel (en referencia a la Seu). Gerona es simplemente la fosilización de una forma autóctona más próxima al étimo latino Gerunda, y también se usó en el catalán escrito hasta la primera mitad del s. XIx, ${ }^{2}$ en competencia con la forma Girona, también medieval pero más moderna. Gerona no es, por tanto, un topónimo de invención castellana, aunque sin duda la pronunciación de este nombre por un catalán y por un castellano siempre fue muy distinta. Lérida fue la evolución autóctona de la Ilerda romana, forma popularizada en todos los reinos vecinos durante los siglos de dominación árabe (Lladonosa, 1977; Coromines, 1989-97: V, 56-67). La forma Lleida se gestó en los territorios cristianos pirenaicos y arraigó en la ciudad al ser conquistada en 1149 por los condados de Barcelona y Urgell. Conviene observar que el catalán medieval desconocía el uso del dígrafo $l l,{ }^{3}$ creación del castellano que adoptaron las lenguas gallega, vasca y catalana, ${ }^{4}$ y por eso antiguamente se escribió Leyda (aunque nunca se pronunció tal como hoy lo leemos). La forma arabizada Lérida

2 Por ejemplo, Josep Ullastra, al tiempo que redactaba su Grammatica cathalána (manuscrita hacia 1753) «per a correctamént parlár i escriurer... la llengua cathalána», se declaraba «oriúndo de la vila de Bañolas, del bisbad de Geróna» (Ullastra, 1980: 1). También se emplea Gerona en la división napoleónica del mariscal Augerau, publicada en el bilingüe (catalán-francés) Diari de Barcelona (2-4-1810).

3 La ll sólo aparece en el catalán medieval en los cultismos: aquellas palabras que el catalán actual grafía $1 \cdot l$. El préstamo ortográfico castellano del uso de la ll ya se había generalizado el s. XVII (Segarra, 1985b: 32-36).

4 Pese a la oposición del principal inspirador de la normativa actual, Pompeu Fabra (Segarra, 1985b: 34). 
pronto desapareció de los textos catalanes bajomedievales, permaneciendo, en cambio, en la lengua castellana. Finalmente, Urgel es conforme con algunas formas latinas medievales. ${ }^{5}$

La situación, de falta de versión castellana para los nombres de las ciudades catalanas, comenzó a cambiar con la supresión del autogobierno catalán. Así, el propio decreto de Nueva Planta de Gobierno para el Principado de Cataluña (9-101715), al establecer la nueva estructura de gobierno territorial (corregimientos) en sustitución de las antiguas veguerías, señalaba como capitales, entre otras: Gerona, Lérida, Villafranca y Vique. ${ }^{6}$ Esta última invención pretendía evitar una terminación consonántica que resulta incómoda en lengua castellana, aplicando la lógica de casos como Mastrique (Maastricht). ${ }^{7}$ En la documentación de carácter más formal Vique sustituyó durante un tiempo el genuino Vich, ${ }^{8}$ y de ahí que sea un topónimo empleado por los cartógrafos catalanes que inmediatamente reflejaron la nueva división de Cataluña en corregimientos: Josep Aparici y el conde de Darnius. Tomás López lo asume en su mapa de 1776, y de ahí lo toman otros como Espinalt (1781). Pero la propia administración acabó abandonando esta forma artificial, ajena a la pronunciación genuina, de modo que en el nomenclátor de 1789 reaparece Vich. ${ }^{9}$

La castellanización aún fue insignificante en los primeros años del nuevo sistema de gobierno, como lo evidencia el primer mapa del Principado editado por un catalán, Josep Aparici, en $1720,{ }^{10}$ en donde sólo aparecen como formas ajenas al catalán: Cherta, Lerida, Tarrasa y Vique (Villafranca designa el corregimiento, pero la localidad aún es Vilafranca). ${ }^{11}$

5 Por ejemplo, Coromines (1989-97: v. VII, 155) cita la forma Urgeldiensis, así como un Urgel fechado en 1290. El cartógrafo Vrients emplea La Seu de Urgel. La presencia de esta terminación en catalán puede tener relación con el mencionado problema de la $l l$.

6 Otra vacilación ortográfica muy «borbónica» pero con menos éxito fue la grafía Manrresa, que Darnius emplea en sus mapas manuscritos.

7 Una lógica habitual en la lengua común: roc/roque, atac/ataque, dic/dique, etc. Esta adaptación también está presente en el valenciano Alberique (por Alberic).

8 La terminación -ch fue general en el catalán anterior a las normas del IEC (1913), aunque diversos gramáticos venían propugnando su supresión: Antoni Font en 1637 (Segarra, 1985) o Ullastra (1980: 267) en 1753. La ch catalana antigua nada tiene que ver, por tanto, con la ch castellana; como advertía el nomenclátor de 1860: «La ch en fin de dicción se pronuncia como c» (Burgueño, 2014: 122).

9 El Prontuario de las ciudades, villas, lugares y parroquias que comprende la provincia de Cataluña (1815) aún conserva Vique.

$10 \mathrm{http} / / /$ cartotecadigital.icc.cat/cdm/singleitem/collection/catalunya/id/3081/rec/1

11 Según Rosselló (2008: 161) los 1.400 topónimos del mapa de Aparici «són escrits amb una ortografia i morfologia correctes per a l'època; amb prou feines n'escapen Gerona, Lerida i Vique.»

Estudios Geográficos, Vol. LXXVIII, 283, pp. 633-656, julio-diciembre 2017

ISSN: 0014-1496, eISSN: 1988-8546, doi: 10.3989/estgeogr.201722 
El nomenclátor de 1789 (Santiago, 1789) permite evaluar el grado de castellanización introducido en la toponimia urbana catalana en el s. XVIII. ${ }^{12}$ La situación era diversa según corregimientos, e incluso en uno mismo se observan diferencias aleatorias. ${ }^{13}$ En el Pirineo y en el corregimiento de Cervera las formas castellanizadas eran prácticamente inexistentes. La castellanización se centró básicamente en tres aspectos: sustitución de ny por $\tilde{n}$ (salvo en final de palabra),${ }^{14}$ hagiotopónimos y villas. La castellanización de advocaciones fue generalizada (en el corregimiento de Manresa casi absoluta) aunque con bastantes excepciones como: San Adriá de Besós, San Just Desvern, San Pere de Ribas, San Quirse de Besora o San Sadurní (d'Anoia). Eso sí, el cambio de sant por san fue total. Algunos corregimientos (Lérida, Tarragona) sustituyeron completamente las vila por villa: Villanueva de la Barca, Villaseca, Villarrodona... En otros, en cambio (Barcelona, Gerona, Manresa y Vic), no se aplicó nunca esa traducción.

Aunque algunas castellanizaciones del s. XviII desaparecen en documentos del primer tercio del XIX, ${ }^{15}$ la mayoría sentaron cátedra. Así quedaron fijadas las formas exóticas: Borjas, ${ }^{16}$ Ceballa del Condado, Ivars de Urgel, Seo de Urgell, Vilella Baja, Villalba, Villanueva y Geltrú o Villanueva del Picat. Las advocaciones más propensas al cambio fueron: Andreu, Esteve, Joan y Miquel.

El célebre Diccionario geográfico de Pascual Madoz, con información referida a la década de 1840 , permite cotejar el avance de la castellanización, especialmente en el 'santoral': ya aparecen denominaciones como San Baudilio (Boi), San Quirico (Quirze), San Lorenzo (Llorenç) o San Saturnino (Sadurní). En la Seu d'Urgell se consolidó la denominación condal Urgel. Como en el caso Seu/Seo, se observa una tendencia a sustituir la terminación -u por -o: Torrelameo y Valencia de Aneo. El único municipio cuyo nombre empieza por $x$, Xerta, se castellanizó — tal como anticipó Aparici- en Cherta.

Por otra parte, la creación de las provincias (1833) dio lugar a la necesidad de distinguir localidades homónimas que anteriormente pertenecían a diferentes

12 Con una laguna notable: en el corregimiento de Tarragona falta la información referida a la antigua veguería de Montblanc.

13 En Barcelona, junto a los impostados San Andrés de la Barca o San Felio de Llobregat se conservan San Climent, San Gervasi o San Boy.

14 En castellano se tendió a pronunciar la terminación -ny como -ni. Por eso el nomenclátor de 1860 advertía «Ny equivale en la pronunciación a la ñ castellana; así Arenys suena Areñs» (Burgueño, 2014: 122).

15 Por ejemplo, en el Prontuario... de 1815 reaparecen las formas Vilanova de Cubellas y la Geltrú e Ivars de Urgell.

16 No conocemos grafías antiguas de les Borges Blanques como Borjas, aunque sí la hay para les Borges del Camp. 
veguerías o corregimientos. Algunos determinantes diacríticos acentuaron la castellanización: de Lérida, del Campo... También la creación de nuevos municipios dio lugar a nombres castellanos: San Carlos de la Rápita, Masías de San Pedro de Torelló, Mora la Nueva ${ }^{17}$ Puerto de la Selva...

\section{BALANCE DE LA CASTELLANIZACIÓN EN ÉPOCA LIBERAL, 1860}

\section{Metodología}

En 1857 se inicia la serie censal contemporánea, que permite verificar los cambios de todo orden (también toponímicos) en la nómina de municipios de España. En particular, el nomenclátor de 1860 fue una colosal aportación al registro de la toponimia, realizada bajo un planteamiento teórico de respeto hacia las lenguas minoritarias (Burgueño, 2014). Esta es la fuente que tomamos como principal referencia para efectuar el balance del grueso de la operación de castellanización de la toponimia municipal catalana bajo el régimen político liberal, por cuanto las modificaciones posteriores fueron relativamente escasas (Burgueño y Aubet, 2016).

Valorar si un topónimo se castellanizó no siempre es tarea fácil, porque, como ya se ha comentado, no sirve como referencia la ortografía catalana actual, vigente a partir de 1913. Hasta entonces el catalán no tuvo una norma aceptada de forma general. En particular hay dos rasgos vacilantes en el tiempo que afectan a buen número de nombres de lugar: la forma del plural (-es vs. -as) y el uso de ny o ñ. Las dos primeras alternativas eran más genuinas, pero su uso no era unánime, y la influencia espontánea del castellano (en los ss. XVI y XVII) dio lugar a un uso creciente de las segundas soluciones. ${ }^{18}$ Por eso, formas toponímicas como Bañolas se consideraban plenamente catalanas a mediados del s. xviII (la emplea Ullastra, natural de esa localidad, en su Grammatica cathalána), ${ }^{19}$ aunque anteriormente la forma más frecuente fuera Banyoles.

17 En catalán Mora de Ebro se habría escrito por entonces de la misma forma. Coromines (1989-97: vol. IV) afirma que Ebro era un mozarabismo autóctono. Aunque aporta testimonios antiguos de Ebre, hoy normativo, en época moderna sólo encontramos Ebro (en Los col-loquis de la insigne ciutat de Tortosa de C. Despuig, 1557, o en el mapa de Vrients, 1604) y Hebro (Andreu Bosch, 1628); Pere Gil, en su Geografia de Catalunya (1600) emplea ambas formas. El primer mapa en el que encontramos Ebre es el de Flos i Calcat (1906).

18 Por ejemplo, Antoni Font, en la primera ortografía catalana, de 1637, defiende el uso de $\tilde{n}$ en lugar de ny (Segarra, 1985a: 49).

19 Este autor era partidario de las dos segundas soluciones. Prefiere el uso de ñ: «axí, no sols es superfluo, sinó també un gran inconvenient escriurer amb ny Cathalúnya, Banyólas [...] tot lo que no esdevé escrivínd ab $n$ ab titlla Cathaluña, Bañolas, etc.» (Ullastra, 1980: 236). 
Por tanto, para afirmar que se produjo una alteración toponímica y, en su caso, una castellanización, es imprescindible cotejar las formas escritas indudablemente catalanas anteriores a la Nueva Planta. En una época en que no existía una determinación oficial que fijara la forma correcta, es natural que la vacilación ortográfica fuese habitual. Por eso no conviene emplear como referencia exclusiva una sola fuente genuina, sino varias. En nuestro estudio utilizamos, entre mapas y listas de localidades, cuatro fuentes principales y tres complementarias, correspondientes todas ellas a los años de 1600 a 1720.

1) Mapa Nova Principatus Cataloniae descriptio, de J. B. Vrients, primer mapa catalán impreso, del que solo se conserva un ejemplar en la Bibliotèque National de París. ${ }^{20}$ Realizado por encargo de la Generalitat y grabado hacia 1604 (Burgueño, 2008, XIII-XVII), su toponimia es inequívocamente catalana (Lleida, Mollerussa, Terrassa, Lo Vendrell...), ${ }^{21}$ aunque al copiar los documentos autóctonos precedentes (hoy perdidos) y en el propio grabado, realizado en Amberes, sin duda se deslizaron errores. La densidad toponímica es alta: encontramos 853 de los municipios estudiados (77\%).

2) Lista de poblaciones de Cataluña ordenada por veguerías, anexo de la obra de Lluís de Peguera Practica, forma y stil de celebrar Corts generals en Catalunya (1632). ${ }^{22}$ Significativamente, el valle de Aran no figura en esta relación, ${ }^{23}$ por lo que hemos buscado estas localidades en otro listado (más deficiente e incompleto) publicada en Perpiñán por Andreu Bosch, en 1628. ${ }^{24}$

20 http://gallica.bnf.fr/ark:/12148/btv1b55005251p/f1.item

21 Según V. Rosselló (2008: 111): «La toponímia, nombrosíssima (1.600 noms, potser), no té res de castellanitzant [...]. La correcció ortogràfica —no la podem jutjar amb normes fabrianes- és mes que satisfactòria, molt superior al que vèiem al cas valencià de 1584» (se refiere al mapa del Reino de Valencia incluido en el Theatrum Orbis Terrarum).

22 «Tercera part en la qual se fa llarga descriptio y compte de tots los llochs, tant Reals com de Barons, de tota la Provincia de Cataluña. Perque assi se veja la grandesa y magnificencia de dita Provincia.»

23 La forma actual de los topónimos araneses sigue la normativa occitana; sólo difiere de la grafía histórica o catalana en la acentuación, el uso de los dígrafos nh y lh (Escunhau, Vielha) y el artículo es para el femenino plural (Es Bòrdes).

24 Summari, index o epitome dels admirables y nobilissims titols de honor de Cathalunya, Rossello y Cerdanya, p. 92-97. Es una lista alfabética de las «Ciutats, castells, viles y llochs de Cathalunya o Comtat de Barcelona, son en numero 1523». 
3) Catalogo de totas las ciutats, vilas y llochs del Principat de Cataluña. Señalanse en que vegaria o sotvegueria son, manuscrito del erudito Pere Serra i Postius (1671-1748), aficionado a recopilar muy diversas informaciones. El documento se conserva en el Arxiu Històric de Protocols de Barcelona y su autor lo debió compilar entre 1711 y $1715 .{ }^{25}$ Además de las localidades de la Cataluña actual, también incluye las de Andorra y la Cataluña francesa. ${ }^{26}$

4) Yndice o relación alfabética de localidades ordenadas por veguerías, en un cuaderno manuscrito de gran formato, que conceptuamos como documento preliminar elaborado en la instauración del Catastro en Cataluña. Se conserva en el Archivo de la Corona de Aragón y se data en 1717. ${ }^{27}$ Únicamente figura en castellano el nombre de algún veguerío: Cervera (Alto y Baxo), Lérida, Villafranca y Vique (en cambio, Girona). No incluye las localidades del valle de Aran, distrito que restó al margen de la Nueva Planta. Nuevamente en este caso hemos recurrido a otro registro manuscrito de la misma índole: el Índice general de los papeles concernientes al Real Tributo de Catastro. ${ }^{28}$

5) Sólo con carácter complementario, hemos empleado también el mapa de Josep Aparici, Nueva descripción geográphica del Principado de Cataluña, de 1720 (Galera, 2006; Burgueño, 2008: XXX-LV). Preparado por el autor años antes de la victoria de Felipe V, ya hemos comentado que su toponimia es inequívocamente catalana. Recurrimos a Aparici únicamente cuando ha convenido añadir otro testimonio ortográfico o bien cuando el geógrafo propone una grafía distinta de la habitual en los documentos coetáneos.

25 Biblioteca. Reserva. Constituye la tercera parte (fol. 185-318) de un volumen manuscrito cuya portada indica «Miscelánea, $\mathrm{n}^{\circ} 82$ ». La autoría se indica en una notación al final: «Soy de Pedro Serra y Postius». Tres aspectos permiten su datación aproximada: el primer documento del libro es una relación cronológica de los diputados que históricamente había tenido la Generalitat de Catalunya, siendo los últimos los designados en el trienio de 1710. Por otro lado, la unidad territorial empleada es la veguería y no el corregimiento, instaurado en 1716-19. Finalmente, en la relación de localidades hay algunas anotaciones posteriores a la redacción general que corresponden al número de casas que recoge el censo de 1719, elaborado por corregimientos y publicado por Josep Iglésies (1974: II). Agradezco a Lluïsa Cases la noticia de la existencia de este manuscrito.

26 En el mismo volumen sigue el documento 4 «Catalogo de las vilas y llochs de Cataluña que están baix del nom de sants y santas» (fol. 319-359) donde encontramos un pequeño número de localidades olvidadas por el autor en la relación anterior.

27 ACA: Hacienda, volúmenes, Inv. 5, $\mathrm{n}^{\circ} 2252$.

28 ACA: Hacienda, volúmenes, TER-P, nº 2071, fol. 85. 
Salvo el mapa de Vrients, el resto de fuentes empleadas recogen la práctica totalidad de las localidades estudiadas, en un porcentaje superior al $93 \%$. La comparación ortográfica la establecemos con todos los municipios catalanes, actuales o bien existentes en 1860: un total de 1.103. ${ }^{29}$ No cabe, por tanto, hablar de una muestra de estudio, ya que se analizan todas las denominaciones oficiales de las localidades catalanas. ${ }^{30}$

Entendemos que el nombre de un municipio contemporáneo (en 1860) presenta un cambio respecto del catalán anterior a 1720 cuando, de la combinación de las 4 o 5 grafías recogidas, no se deriva fácilmente la forma oficializada en época liberal. Así por ejemplo, Figueras es la forma empleada por Peguera y por el Catastro, aunque Vrients y Aparici recogen Figueres y Serra Postius emplea Figeras. ${ }^{31}$

Es innegable que el catalán ya se había deslizado hacia soluciones castellanas antes de 1714, pero lo que no puede afirmarse es que esta deriva sea imputable a la presión ejercida por el aparato del Estado borbónico ni por el liberal. ${ }^{32}$ Así, si Bañolas se emplea en todo el s. xIX como denominación oficial, la causa no es una castellanización súbita e impuesta por una administración centralizada, sino un proceso endógeno del catalán que se produce - eso sí- bajo la influencia del castellano como lengua de prestigio. ${ }^{33}$ Vrients recoge Bañoles, Peguera y Aparici se decantan por la forma Banyoles, hoy oficial, en tanto que Serra Postius y el documento catastral emplean el híbrido Banyolas.

Hasta que el catalán no logre elaborar unas soluciones ortográficas estandarizadas y depuradas de la influencia castellana, la mayoría de las formas toponímicas oficiales serán percibidas como perfectamente catalanas y

29 Dejamos al margen del cómputo 11 municipios inexistentes el s. xviII (que elevarían el cómputo a 1.114), pero sí hemos incluido aquellos creados el s. XIx en los que la castellanización es palmaria (Puerto de la Selva, San Carlos de la Rápita...).

30 Las tablas con toda la información recogida y su clasificación podrán encontrarse en el Repositori Obert UdL: https://repositori.udl.cat

31 Un caso dudoso es el de Terrassa. Consideramos que Tarrasa es una castellanización que ya emplea Aparici porque todos los testimonios anteriores lo escriben con ss. En cambio, el paso de $e>a$ ya se observa en la lista de Postius.

32 Por ejemplo, Mila Segarra (1985b: 63) sitúa a finales del s. Xvil la plena sustitución del plural -es por -as, mientras que en el siglo anterior se empleaban ambas formas.

33 Debe advertirse que en la mitad oriental de Catalunya la terminación plural -as es conforme con la pronunciación, al existir neutralización fonética de las vocales $a$ i $e$. Es significativo que un gramático del s. XviI natural de Banyoles propugnara el uso del artículo las y de la forma plural «donas, casas, cosas i no dones, cases, coses» (Ullastra, 1980: 49). 
autóctonas. ${ }^{34}$ La diferencia entre forma oficial y forma puramente catalana se irá acentuando en la medida que se geste una normativa moderna durante la Renaixença, ${ }^{35}$ y ya de forma definitiva, con la traslación de las normas del IEC a los municipios durante la II República (Generalitat de Catalunya, 1933). Así, la toponimia oficial bajo el franquismo (Bañolas, Figueras, Montblanch, Moyá, Vich...) progresivamente dejó de ser percibida como propia para pasar a ser vivida como otra imposición más de un régimen beligerante ante cualquier tentativa de dignificar y promover la lengua catalana. Se convirtieron en formas supuestamente «castellanas», aunque la mayoría no lo eran.

\section{Resultados}

\section{Continuidad}

Tres cuartas partes de los topónimos municipales (836) no experimentaron una modificación sustancial respecto a las grafías anteriores a la Nueva Planta. ${ }^{36}$ El porcentaje más alto de continuidad se registra en la provincia de Tarragona (82\%) y el más bajo en la de Barcelona (70\%). Las cifras son suficientemente altas como para negar la existencia de una castellanización masiva e indiscriminada de la toponimia municipal catalana. La mayor parte de los genéricos no se tradujeron, en particular el abundante Castell, pero tampoco otros términos que hubiera sido fácil traducir al castellano caso de haber existido una política más beligerante contra la lengua propia del país: Aigua, Bisbal, Coll, Molins, Palau, Pla, Pobla, ${ }^{37}$ Pont, Prat, Puig o Vall. Incluso la mayoría de vilas no mutaron en villas. Tampoco se deformó de forma grotesca la ortografía y fonética catalana, para aproximarla a la castellana, como se hubiera

34 Desde luego no lo eran todas. Por ejemplo, el nomenclátor de 1860 de la provincia de Barcelona añade a la forma oficial un centenar de formas catalanas equivalentes a las denominaciones oficiales (Burgueño y Aubet, 2017).

35 Significativamente con la reivindicación del plural -es por parte de Marià Aguiló (Segarra, 1985a: 183).

36 No tenemos en cuenta la ausencia de acentuación. El catalán medieval no acentuaba; el uso del acento se fue incorporando de forma asistemática, principalmente por influencia de la norma castellana, aunque Aparici, como los autores del s. XVII (Segarra, 1985a) siempre empleaba el acento grave ('). Tampoco atendemos a la separación o aglutinación de los componentes del topónimo, ni a la presencia o ausencia de artículo cuando este falta en alguna de las fuentes no cartográficas consultadas (en los mapas antiguos casi siempre se obvian los artículos).

37 Las siete Pobla catalanas siempre conservaron su genérico. Esta es una diferencia significativa con el caso valenciano, donde las seis Pobla se tradujeron a Puebla. 
hecho, por ejemplo, cambiando siempre -ll por -l, sustituyendo toda ny por $\tilde{n}$ o añadiendo una vocal final postiza para evitar las «incómodas» terminaciones consonánticas, como se intentó sin éxito con Vique. Efectivamente, es remarcable la ausencia, en Cataluña, de la castellanización mediante una -e paragógica, profusamente usada en Valencia (Rosselló, 2004: 180): Bocairente, Calpe, Carcagente, Elche, Onteniente...

\section{Cambios leves}

Un reducido número de topónimos experimentó una ligera modificación respecto de los precedentes autóctonos estudiados. En 22 casos parecen simples vacilaciones ortográficas sin mayor trascendencia fonética: alternancia inocua de $v$ i $b$ (Alcover, Vilallobent), ${ }^{38} o>u$ (Colera, Castellfollit), ${ }^{39}$ añadido de -t en La Quar, ${ }^{40}$ cambio de Urx (o Urg) por Urtg i duplicación de $r$ en Vilarodona. Cabe añadir un cambio semántico de menor entidad: del tradicional Aynet y Besan a Aynet de Besan. ${ }^{41}$

\section{Cambios leves pero relevantes}

En un número superior de modificaciones de poca entidad (57) se observa cierta intención castellanizadora. Un ejemplo frecuente es el uso de $\tilde{n}$ allí donde todos los antecedentes estudiados presentaban ny (Liñola, Riudecañas). No lo consideramos una castellanización flagrante porque su uso no tiene consecuencias fonéticas y porque en los ss. XVIII y xix la ñ no se consideraba una grafía ajena al catalán, al contrario, diversos autores propugnaban su uso exclusivo. ${ }^{42}$ Un caso similar es la mutación de la terminación de plural -es por -as (Folgarolas, Pardinas), ya comentada. ${ }^{43}$ La terminación -ja pasa en ocasiones a -ija (Torroija, Ribarroija), sin que ello implique —más bien al

38 En la mayor parte de Cataluña se ha perdido la diferencia fonética entre $b$ y $v$.

39 En el catalán oriental la $o$ y la $u$ se aproximan fonéticamente (se neutralizan).

40 Posible paragoge. La Mancomunitat de Catalunya consideró correcto La Quart en 1918.

41 Aunque sea un cambio banal no es lógico mantenerlo, pues Ainet no depende para nada de Besan.

42 Ullastra (1980: 207) llega a propugnar su utilización en final de palabra: Montseñ.

43 También contabilizamos el añadido diacrítico de las Monjas aplicado a Vallbona como castellanización de menor entidad (alejada de la pronunciación local: de les Monges). Con todo, en el catalán de la época posiblemente se habría escrito del mismo modo, como testimonia el diccionario de Lavernia (1840). 
contrario- una modificación más cómoda en castellano. Una castellanización ortográfica más evidente es la pérdida de ss, pasando a s (Llusanés, Mollerusa) o a $c$ (Cabacés), ${ }^{44}$ o el cambio de $z$ o $s$ por $c$ (Doncell), aunque nuevamente todo ello no implicaba cambio fonético en catalán. Igualmente, el cambio de Sant por San (por ejemplo San Jordi Desvalls) normalmente no suponía una distorsión fonética (esa -t sólo se pronuncia precediendo vocal, como en Sant Antoni), aunque sí representaba una clara castellanización ortográfica. También incluimos en esta categoría el cambio de $v$ por $b$ cuando parece responder a la diferente grafía del término expresado en catalán y en castellano: Tabérnolas (por taberna) o Viladecaballs (caballo). La pérdida de la h- (Hurtó>Ortó), sin ser castellanización, sí es un cambio sensible. Finalmente, también contabilizamos un cambio del artículo femenino plural les por las (Las Irlas). ${ }^{45}$

El nomenclátor de 1860 acentuó los topónimos de acuerdo con la norma entonces vigente en castellano «con la advertencia de que en caso de duda vale más pecar por exceso que por omisión de acentos, para evitar dudas y equivocaciones». ${ }^{46}$ Generalmente la acentuación se hizo perfectamente acorde con la pronunciación catalana de los topónimos, con la excepción de diversas poblaciones de la provincia de Lérida, en la que se estableció una acentuación absurda; los errores más graves que perduraron —algunos durante décadasfueron: Albí, Anéu, Aransá, Aréo, Arrés, Artiés, Cerní, Guíxes, Novés, Óden, y Pedrá. ${ }^{47}$ Todos estos casos los contabilizamos como modificaciones relevantes.

\section{Depuración}

Existen otros muchos cambios leves que difícilmente podemos conceptuar como castellanización o deformación porque esa innovación supuso, al fin y al cabo, una aproximación a la grafía oficial actual, generalmente determinada por el IEC. ${ }^{48}$ Aparentemente son fruto de una tarea de depuración de la forma

44 Aunque esta es la forma oficial actual, no consideramos que fuese una mejora del topónimo, ya que el IEC propugna Cabassers, en contra del criterio del Ayuntamiento.

45 Aunque les ya era excepcional a principios del s. xviII: Postius recoge 4 casos frente a 36 las. Hacia 1750 el gramático Ullastra (1980: 46) propugnaba el uso en catalán del artículo femenino las.

46 «Instrucción para llevar a efecto la rectificación y complemento del Nomenclátor de los pueblos de España», 5-I-1859, cuya redacción atribuimos principalmente al geógrafo Fermín Caballero, sensible ante la cuestión toponímica (Caballero, 1834; Burgueño, 2014: 29 y 91).

47 En la provincia de Barcelona, en el nomenclátor de 1860 (pero no en el censo) figura Capéllades, pero el censo de 1877 lo corrigió en Capelládes.

48 Aunque algunos ayuntamientos rechazan el criterio normativo del IEC y prefieren mantener una grafía arcaica. 
escrita correcta, quizás debida a la consulta de documentación eclesiástica, más fiel a la etimología. ${ }^{49}$ Paradójicamente, en algún caso pudo tratarse de castellanizaciones, como al cambiar Eulària por Eulalia o Cicilia por Cecilia, que al fin y al cabo resultaron coincidentes con las formas catalanas admitidas hoy día (convenientemente acentuadas). Fuera como fuere, observamos 74 casos (cerca del $7 \%$ del total) de mejoría del topónimo, entendiendo por ello su acercamiento a la forma oficial actual.

Esta tipología es muy diversa. En algunos casos se observa un cambio radical del nombre de la población: Almçarra $>$ La Musara, Derdola $>$ Olérdola, Estarach $>$ Estrach, Ferreginals $>$ Freginals, Fontrubia $>$ Fontrubí, Lovan $>$ Olban, Moster $>$ Almoster, Samalcoreix $>$ Masalcorreig, Santa Arada $>$ Senterada, Valldellos>Vandellós, Vellusell> Vilosell y Viá>Aviá. Es frecuente el cambio de y a $i$ (los antiguos Alguayre, Bellcayre, Sapeyra...). Igualmente la inclusión de una $i$ entre vocal y $x$ : Aleixar, Creixell, Freixenet, Preixana, Vinaixa. Se registra igualmente la recuperación de la $t$ en mont- (Montgrí, Montmell, Montreal) o la de alguna otra letra etimológica imperceptible en la pronunciación local (Caldès $>$ Calders, Dosques $>$ Dosquers, Dorrius $>$ Dosrius, Rimors $>$ Riumors, Vallobrega $>$ Vall-llobrega). Son diversos los casos de corrección de una vocal a en $e,^{50}$ como en el paso de torra a torre, y también en: Balenyá, Begudá, Borredá, Cardedéu, Farrera, Llesuy, Tarrés, Teyá, Tredós y Vallcebre. La grafía del plural se corrige en Capsanes, Menarguens y Saldes. Otros casos de rectificación de una o dos letras son: Bellmont>Bellmunt, Calonja $>$ Calonge, Cellent $>$ Sallent, Cevís $>$ Civís, Collsespina $>$ Collsuspina, Esparaguera $>$ Esparraguera, Orta $>$ Horta, Salrá>Celrà y Vinbodí>Vimbodí.

\section{Castellanización de hagiotopónimos}

La forma más primaria de castellanización (como en cualquier traducción de un nombre de lugar) se aplica a las advocaciones o hagiotopónimos. Este mecanismo se observa en 77 municipios ( $7 \%$ del total). La situación es netamente diferente entre provincias. En Lérida y Tarragona únicamente se tradujo un caso en el nomenclátor de $1860,{ }^{51}$ manteniendo el resto su forma

49 No hemos empleado ninguna fuente eclesiástica en este estudio, pues ello implicaba localizar la fuente idónea para cada una de las nueve diócesis.

50 La confusión de $a$ i $e$ a menudo es consecuencia de la neutralización de estas dos vocales en el catalán oriental.

51 Aunque lo contabilizamos, San Carlos de la Rápita no es propiamente una traducción, sino el nombre original castellano de la nueva población fundada bajo el reinado de Carlos III. 
plenamente catalana (Sant Antolí, Sant Jaume, Sant Martí, Sant Pere, Sant Vicens...). ${ }^{52}$ Fuera de estas provincias, la $t$ final del genérico únicamente se conservó en el gerundense Sant Aniol (Nadal y Urteaga, 2012). En las provincias de Gerona y Barcelona se tradujeron la gran mayoría de los hagiotopónimos (al margen, claro está, de los diversos que presentan la misma forma en ambas lenguas, como María, Perpetua o Salvador). ${ }^{53}$ Resulta más breve indicar las excepciones que resistieron al cambio de lengua. Generalmente se trata de nombres de muy forzada traducción: Celoni, Coloma, Cugat, Feliu (pero $S$. Felío de Codinas), Mori, Pol, Privat y Pau (santa). Excepcionalmente se salvó el San Sadurní de la provincia de Gerona (no los dos de Barcelona) y San Boy de Llusanés (a diferencia de su homónimo del Llobregat). Ejemplos de traducción muy forzada son: Iscle en Acisclo, Cebrià en Cipriano y Quirze en Quírico.

\section{Castellanización plena o deformación}

Finalmente, detectamos 37 casos de deformación o castellanización manifiesta. Se trata de una minoría (un 3,3\% del total) pero su número absoluto es elevado en la provincia de Lérida (21). La mayoría son villas (Villalba, Villafranca, Villanueva), pero este concepto no se traduce nunca en la provincia de Gerona y en las otras hay numerosas excepciones (Viladecans, Vilanova del Camí, Vilanova de Prades, Vilanova de Meyá...). En general no son una novedad del régimen liberal, sino traducciones heredadas del s. XVIII o principios del xIX. Algunas corrupciones (no propiamente castellanizaciones) de nuevo cuño fueron: Aréo, Arsequell, Asentin (la Sentiu), Bagerque, Cogull, Sampedor, Tivenis, ${ }^{54}$ Valéncia de Aréo y Vilech. ${ }^{55}$

Las castellanizaciones netas que se introdujeron entre 1842 (Madoz) y 1860 fueron únicamente cinco: Castellón de Ampurias, Castillo de Aro, Mollet cerca de Peralada, Valle de Castellbó y Villalba Saserra, a las que cabe sumar el añadido de Lérida en Benavent y Montoliu.

52 La castellanización de los hagiotopónimos tarraconenses es posterior (censo de 1910).

53 El redactor del nomenclátor de Gerona de 1860 era explícito en el criterio seguido: «Se han escrito en castellano aquellos nombres de Santos que se pronuncian ya, como se escriben en este idioma; pero no los que siguen nombrándose en el dialecto catalán, ni aquellos otros que carecen de equivalencia en castellano, como San Medir, Sant Aniol.» (Burgueño, 2014: 139).

54 Esta deformación no aparece propiamente en el nomenclátor de 1860 , sino en su fe de erratas.

55 Barbará ya figura con esta grafía en 1815. 
TABLA 1

\begin{tabular}{l|r|r|r|r|r|r|r|r|r|r}
\hline \multirow{2}{*}{ Tratamiento de los topónimos } & \multicolumn{2}{|c|}{ Barcelona } & \multicolumn{2}{|c|}{ Girona } & \multicolumn{2}{c|}{ Lleida } & \multicolumn{2}{c|}{ Tarragona } & \multicolumn{2}{|c}{ total } \\
\cline { 2 - 14 } & \multicolumn{1}{c|}{$\#$} & \multicolumn{1}{c|}{$\%$} & \multicolumn{1}{c|}{$\#$} & \multicolumn{1}{c}{$\%$} & \multicolumn{1}{c}{$\#$} & \multicolumn{1}{c}{$\%$} & $\#$ & $\%$ & $\#$ & $\%$ \\
\hline Depuración & 22 & 6,6 & 12 & 4,8 & 25 & 7,6 & 15 & 8 & 74 & 6,7 \\
\hline Continuidad & 233 & 70,2 & 196 & 77,8 & 252 & 76,4 & 155 & 82 & 836 & 75,8 \\
\hline Cambio leve o neutro & 3 & 0,9 & 9 & 3,6 & 9 & 2,7 & 1 & 0,5 & 22 & 2 \\
\hline $\begin{array}{l}\text { Cambio menor castellanizador o } \\
\text { modificación de cierta entidad }\end{array}$ & 12 & 3,6 & 14 & 5,5 & 22 & 6,7 & 9 & 4,8 & 57 & 5,2 \\
\hline Castellanización de la advocación & 58 & 17,5 & 17 & 6,7 & 1 & 0,3 & 1 & 0,5 & 77 & 7 \\
\hline Castellanización o deformación & 4 & 1,2 & 4 & 1,6 & 21 & 6,3 & 8 & 4,2 & 37 & 3,3 \\
\hline \multicolumn{1}{c}{ Municipios } & 332 & 100 & 252 & 100 & 330 & 100 & 189 & 100 & 1.103 & 100 \\
\hline
\end{tabular}

\section{Afrancesamiento de los nombres de los municipios de la Catalunya del Nord}

La anexión a Francia de una parte del territorio catalán, acordada en el Tratado de los Pirineos (1659), permite cotejar el proceso de castellanización con el de afrancesamiento. Las fuentes históricas empleadas para establecer las formas catalanas en este territorio en buena parte han sido las mismas: el mapa de Vrients (ca. 1604), la lista de Lluís de Peguera (1632), el catálogo de Pere Serra i Postius (ca. 1712) y complementariamente (casi únicamente para la Cerdanya francesa) el mapa de Aparici (1720). A estas añadimos las listas de poblaciones de los antiguos condados de Rosellón y Cerdaña publicadas por Andreu Bosch en Summari, index o epitome dels admirables y nobilissims titols de honor de Cathalunya, Rossello y Cerdanya (Perpiñán, 1628). ${ }^{56}$

Lluís Basseda, estudioso de la toponimia de la Catalunya del Nord, también aborda las formas de afrancesamiento (Basseda, 1990: 20-26 y 279-285), llegando a la conclusión que aproximadamente la mitad de los topónimos principales se han afrancesado.

Nosotros hemos estudiado 201 nombres de comunnes del departamento de los Pirineos Orientales. ${ }^{57}$ En consonancia con el marco temporal de nuestro estudio anterior, también hemos tenido en cuenta las comunas existentes a mediados del s. xix pero agregadas a otras posteriormente (Pelissier, 1986). En el caso francés la comparación del topónimo catalán antiguo puede

56 En las páginas 81-82 (Rossellón y Vallespir), 85-86 (Cerdanya) y 89 (Conflent y Capcir).

57 Se exceptúa la comarca de la Fenolleda, de lengua propia occitana, que no formaba parte de las tierras españolas agregadas a Francia en 1659. 
establecerse directamente con la forma oficial actual, ya que Francia no ha admitido prácticamente ningún topónimo en catalán normativo, salvo en un caso, en el cual la forma afrancesada resultaba ofensiva. ${ }^{58} \mathrm{Al}$ igual que en la Cataluña hispánica, por lo general prescindimos de valorar cambios menores: de acentuación, uso de mayúsculas, apóstrofo y guiones. Tampoco consideramos relevante la aglutinación (la Sella>Lasseille) o su contrario (Entrevalls $>$ Entre-Valls). De este modo, contabilizamos solo un tercio de topónimos que presentan continuidad en relación a las formas anteriores al proceso de afrancesamiento.

De manera análoga al caso español, podemos considerar cambios menores:

- cambio del grup -ig en -itg (Molitg),

- cambio de $v$ por $b$ (Cerbère),

- cambio de -i por -y (Py),

- vacilación entre u y $o$ (Amont, Sorède),

- vacilación entre las opciones ss, s, ç y $z$,

- ubicación de $h$ en posición desplazada respecto de la que nosotros hemos podido documentar. ${ }^{59}$

Pero estas modificaciones de menor entidad casi siempre se acompañan de otras mutaciones de mayor calado y afrancesamiento, de manera que únicamente registramos seis topónimos que presentan una alteración leve: Corsavy, Enveitg, Py, Thuès-Entre-Valls, Thuir y Urbanya.

Evidentemente la acentuación se rige por la norma del francés. Pero esta cuestión no la consideramos relevante (en determinados contextos es imperceptible) salvo cuando la tilde sitúa la fuerza de voz en una sílaba distinta de la genuina catalana: Canohès, Pézilla, Théza... Este tipo de alteración está presente (sin compañía de otras deformaciones) en solo tres topónimos (Alénya, Caldégas y Céret) ${ }^{60}$ y constituye el único supuesto de alteración que hemos conceptuado como de tipo medio.

Casi todo el santoral se vertió al francés (únicamente se salvó Marsal), así como los genéricos saint y sainte, en todos los casos. Son 13 los municipios que presentan un galicismo centrado exclusivamente en el hagiotopónimo.

58 El catalán La Clusa (Alta y Baixa) se afrancesó en L’Écluse, que en francés significa la enclusa; en 1984 se adoptó una forma correcta: Les Cluses.

59 Basseda (1990) aporta otros testimonios de grafías antiguas que explican opciones de oficialización aparentemente arbitrarias. No obstante, únicamente tomamos como referencia las fuentes señaladas para no alterar la metodología y facilitar la comparación con el caso español.

60 En un solo caso, Néfiach, el nombre de lugar presenta a la vez una depuración (e etimológica) y una deformación por acento. Hemos priorizado la primera circunstancia. 
El afrancesamiento y deformación de los topónimos de la Catalunya del Nord, presenta múltiples formas:

- Supresión de -a final átona (Ballestavia>Baillestavy) o bien substitución por -e (LAlbère, La Cabanasse, Corbère, Eyne...) o por -es (Prades, Formiguères).

- Añadido de - $n$ tras - a tónica (Perpignan).

- Sustitución de los artículos lo/el por le (Le Perthus, Le Soler) ${ }^{61}$ y los/els por les (Les Angles).

- Traducción de Puig por Puy (Puyvalador, Puymorens), Torre (o Tor) por Tour, Vall por Val (Valcebollère), -llonga por -longue (Serralongue, Villelongue), así como las Villefranche y Villeneuve.

- Substitución de o y u por ou, muy frecuente: Angoustrina, Boule, Coustouges, Toulouges, Boulou...

- Interposición de una i en los grupos ull (Brouilla, Fuilla) y ell (Corneilla, Odeillo, Oreilla, Railleu...).

- Deformación (no traducción) de Castell en Casteil o Castel.

- Pérdida de $l l$ (Estagel, Belpuig).

- Cambio de las terminaciones -es en -ies (Caudiès) y -ca en -que (Roque, Salanque).

- Interposición de una $h$ sin antecedentes (Canohès, Le Perthus, Théza).

- Añadido de una expresión netamente francesa: -sur-Mer, -sur-Tech, -enRoussillon, Amélie-, -des-Albères, -de-Cerdagne, -la-Rivière, -le-Château, -des-Fontaines, -de-la-Commanderie, -Bas-, -les-Bains, así como uso de la conjunción copulativa et. Deben añadirse dos topónimos creados bajo dominio francés: Bourg-Madame y Mont-Louis.

- Elisión de la preposición de y yuxtaposición de dos elementos del topónimo: Corbère-les-Cabanes, Saint-Jean-Pla-de-Corts.

- Cambio del dígrafo catalán ny por gn (Perpignan).

- Duplicación de las consonantes $n$ y $t$ : Olette, Targassonne.

- Supresión, agregación o modificación de vocal sin causa clara: Marquixanes, Périllós, Peyrestortes, Souanyas. Una deformación compleja enigmática es el cambio de la terminación ull por -vel en Tautavel.

Lógicamente algunos topónimos pueden presentar diversas de estas alteraciones: Sallagosa $>$ Saillagouse, Trullas $>$ Trouillas o el propio Perpignan. La mitad de los nombres de municipios de la Cataluña francesa (51\%) sufrieron

${ }_{61}$ Este motivo permite incluir Le Barcarés (segregado en 1929), pese a que no exista una grafía catalana histórica. 
una deformación mayor o afrancesamiento del tipo indicado. A este porcentaje cabe sumar la traducción de los nombres de santos y el desplazamiento de acentos, con lo que el afrancesamiento total supera el $59 \%$.

Finalmente también se registra un reducido número de casos (10) cuya alteración gráfica, vista en perspectiva histórica, supuso una depuración de la grafía, acercándola al catalán normativo actual. ${ }^{62}$ Por ejemplo, al adoptar el plural en -es (Dorres, Torderes) ${ }^{63}$ recuperar una vocal etimológica que se había modificado por neutralización (Néfiach), añadir $e$ - antes de s- (Spira>Espira), sustituir $y$ por $i$ (Clairà) o corregir $n p$ en $m p$ (Bompàs).

TABLA 2

\begin{tabular}{l|c|c}
\hline \multicolumn{1}{c|}{ Tratamiento de los topónimos } & $\#$ & $\%$ \\
\hline Depuración & 10 & 5 \\
\hline Continuidad & 66 & 32,8 \\
\hline Cambio leve o neutro & 6 & 3 \\
\hline Acentuación desplazada & 3 & 1,5 \\
\hline Afrancesamiento de la advocación & 13 & 6,5 \\
\hline Afrancesamiento o deformación $\quad$ Communes & 103 & 51,2 \\
\hline \multicolumn{2}{c|}{$\quad 201$} & 100 \\
\hline
\end{tabular}

\section{Afrancesamiento en los mapas de Cataluña}

La cartografía de Cataluña publicada en París evidencia el mismo grado de afrancesamiento y deformación toponímica que experimentó el Rosellón bajo administración francesa. Debe recordarse que la intervención de tropas francesas en suelo catalán fue reiterada en diversas ocasiones entre 1640 y 1827 , lo que les permitió recopilar y elaborar información de primera mano sobre el terreno.

Llegamos a esta conclusión tras verificar cinco mapas franceses muy representativos:

- Sanson d'Abbeville, Principauté de Catalogne, $1660 .{ }^{64}$

- Beaulieu, Carte de la Principauté de Catalogne, ca. 1668. ${ }^{65}$

62 La denominación catalana de las comunas de los Pirineos Orientales se recoge en el Nomenclàtor toponímic de la Catalunya del Nord, publicado por el IEC y la Universitat de Perpinyà (2007).

63 También sucede el caso inverso: Soanyes se convirtió en Souanyas.

${ }^{64} \mathrm{http}: / /$ cartotecadigital.icc.cat/cdm/singleitem/collection/catalunya/id/1769/rec/1

$65 \mathrm{http}: / /$ cartotecadigital.icgc.cat/cdm/singleitem/collection/atles/id/687/rec/109. Complementamos este mapa de pequeño formato con las restantes obras de Beaulieu que representan las poblaciones estudiadas, pues las vacilaciones son frecuentes; por ejemplo: Barcelone, Barçelone, Barcelonne. 
- Michel-Antoine Baudrand, La Principaute de Catalogne et le comte de Roussillon, $1703 .{ }^{66}$

- Roussel, Carte generale des Monts Pyrénees, $1730 .{ }^{67}$

- Louis-Gabriel Suchet, Théatre des opérations de l'Armée d'Aragon, $1828 .{ }^{68}$

En una muestra de 53 poblaciones, ${ }^{69} \mathrm{el}$ porcentaje de nombres afrancesados o simplemente distorsionados (aplicando la misma metodología que en los casos anteriores) varía entre el 35\% de Sanson y el 70 \% de Baudrand. Una proporción mucho más elevada que la registrada en los mapas de filiación catalana. Como contrapunto calculamos los valores referidos a los ya mencionados mapas de Vrients y Aparici, así como el Mapa del Principado de Cataluña de Tomás López (Madrid, 1776) ${ }^{70}$ y el Mapa de Catalunya del pedagogo Francesc Flos i Calcat, primero de gran formato rotulado íntegramente en catalán (Barcelona, 1906). ${ }^{71}$

TABLA 3

\begin{tabular}{l|c|c|c}
\hline \multirow{2}{*}{ Mapa } & \multirow{2}{*}{$\begin{array}{c}\text { Topónimos } \\
\text { estudiados }\end{array}$} & \multicolumn{2}{|c}{ Distorsión, afrancesamiento o castellanización } \\
\cline { 3 - 4 } & 49 & Número* $^{*}$ & $\%$ \\
\hline Sanson, 1660 & 38 & 17 & 34,7 \\
\hline Beaulieu, 1668 & 52 & 24,5 & 64,5 \\
\hline Baudrand, 1703 & 49 & 36,5 & 70,2 \\
\hline Roussel, 1730 & 49 & 25 & 51 \\
\hline Suchet, 1828 & 53 & 22 & 44,9 \\
\hline Vrients, 1604 & 53 & 7,5 & 14,1 \\
\hline Aparici, 1720 & 53 & 6,5 & 12,3 \\
\hline López, 1776 & 53 & 10 & 1,9 \\
\hline Flos i Calcat, 1906 & & 1 & $y$ \\
\hline
\end{tabular}

* Los decimales obedecen a que algún topónimo aparece rotulado dos veces, una correcta y otra no.

66 http://cartotecadigital.icgc.cat/cdm/singleitem/collection/catalunya/id/1169/rec/1

$67 \mathrm{http} / / /$ cartotecadigital.icgc.cat/cdm/singleitem/collection/europa/id/2672/rec/2

$68 \mathrm{http}: / /$ cartotecadigital.icgc.cat/cdm/singleitem/collection/espanya/id/2681/rec/11

69 Las 43 capitales de comarca más: Agramunt, Bellpuig, Bellver, Camprodon, Cardona, Castellfollit, Hostalric, Palafrugell, Ribes de Freser y Roses.

$70 \mathrm{http}: / /$ cartotecadigital.icgc.cat/cdm/singleitem/collection/catalunya/id/1774/rec/3

$71 \mathrm{http}: / /$ cartotecadigital.icgc.cat/cdm/singleitem/collection/catalunya/id/1576/rec/1. Este mapa presenta una única forma errónea según nuestro criterio: la pérdida de la ss en Mollerussa carece de precedentes anteriores a 1720. En cambio, sí consideramos formas autóctonas: Banyolas, Borjas d'Urgell, Figueras, Ribas y Rosas, aunque hoy serían inaceptables. 
El afrancesamiento alcanza topónimos inalterados en los mapas elaborados al sur de los Pirineos: Agramont, Balaguier, Belpouche, Bellevert, Castelfolit, Mataros, Puyçerda, Reous, Ripouil, Rose, Sabadel, St. Feliou, Ste. Colombe de Farnes, Terraga (Tàrrega), Tremps, Vails, le Vendrel, Viela... En particular, se evidencia la aversión del francés ante la terminación en a: Amposte, Barcelone, Bergue, Cardone, Cervere, Gironne, Ygualade, Manrese, Solsone, Taragone, Tortose... Otras deformaciones observadas son: Lo Bisbal, Bagnoles, Borje Blanches, Campredun, Ostalrie, Figuieres, Monblancq, Palagrugel, St. Estienne de Rives, Tarasa, Urgel, Ville Franche y Villeneuve.

\section{Algunas CONCLUSIONES... Y UNA REFLEXIÓN}

La toponimia genuina catalana sufrió un proceso de castellanización a partir de la supresión del autogobierno del país, de resultas de la derrota de 1714. Sin embargo, este proceso tuvo una extensión e intensidad muy inferior al afrancesamiento de la toponimia que sufrió la parte de Cataluña incorporada a Francia en 1659. Sumando diferentes formas de castellanización (o simple deformación) estas únicamente afectaron el 15\% de los municipios catalanes. Aunque faltan estudios para otras zonas de la Península que nos permitan establecer comparaciones objetivas, está claro que en el caso valenciano el porcentaje fue muy superior (Rosselló, 2004: 179). ${ }^{72}$

La mayoría de los municipios catalanes emplearon durante la época contemporánea denominaciones que fueron el resultado evolutivo y espontáneo del uso y forma de escribir el catalán antes de 1720 (una espontaneidad no exenta, claro está, de la influencia del castellano, lengua de prestigio normativizada en fecha temprana).

En la Cataluña francesa, las diversas formas de deformación y afrancesamiento toponímico alcanzaron el $59 \%$ de las communes de la zona catalanófona del departamento de los Pirineos Orientales. La actitud del Estado ante la toponimia local tuvo, por tanto, una intención deliberadamente asimiladora. La cartografía francesa referida a la Cataluña meridional evidencia que la misma suerte de afrancesamiento que sufrió el Rosellón se habría aplicado al sur del Pirineo.

72 Concretamente afirma que el $43 \%$ de las cabezas de municipio en la zona de habla catalana presentan una forma correcta, «que podríem estendre a prou més de la meitat si prescindíem de detalls d'accentuació, hacs o articles». El arraigo y diversidad de formas toponímicas castellanas en el territorio valenciano sin duda tiene relación con la vecindad de poblaciones castellanófonas, que comparten desde siempre el mismo Reino, algo que no sucede en Cataluña. 
FIGURA 1

EVOLUCIÓN DE LA TOPONIMIA MUNICIPAL: DE LA FORMA CATALANA A LA OFICIAL EN EL S. XIX

\begin{tabular}{|l|c|}
\hline Catalunya (España) & 74 \\
\hline Depuración & 836 \\
\hline Continuidad & 22 \\
\hline Cambio leve & 57 \\
\hline Cambio medio & 77 \\
\hline Cambio de hagiotopónimo & \\
\hline Deformación, forma & 37 \\
\hline foránea & \\
\hline
\end{tabular}

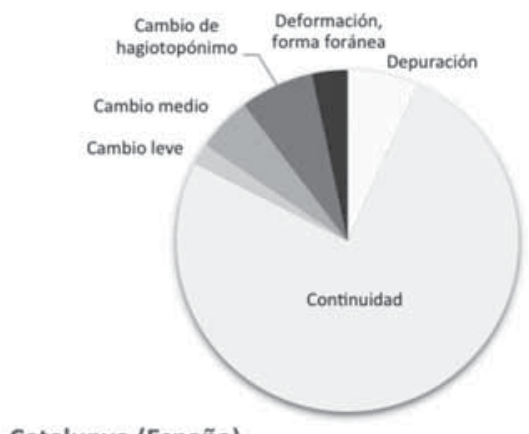

Catalunya (España)
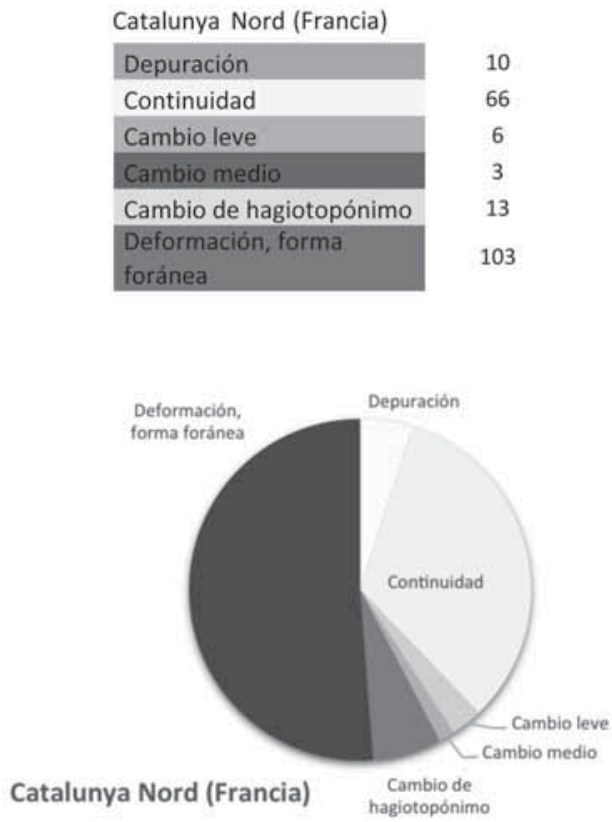

La cuestión toponímica a menudo se convierte en arma arrojadiza. Cabría esperar que la construcción del Estado autonómico hubiera dado lugar al máximo respeto y uso de la toponimia propia de cada territorio, sin renunciar por ello al uso de las formas incorporadas al castellano desde hace siglos, como Gerona, Lérida o Perpiñán. Ciertamente, Cataluña tiene una sola forma oficial de los topónimos, tal y como recomiendan los lingüistas (Mujica, 2010: 77). ${ }^{73}$ Así lo entienden también los organismos del Estado (INE, IGN). Se cumple, pues el principio de univocidad, de asignación de un único nombre normalizado a cada entidad geográfica, para evitar la ambigüedad:

«Para una comunicación clara, inequívoca y eficaz debe existir de cada nombre de lugar una forma única declarada oficial, pues el uso arbitrario de un mismo topónimo bajo diversas variantes podría dar lugar a todo tipo de confusiones y conflictos». (Ruhstaller y Gordón Peral, 2013: 34)

\footnotetext{
73 Cita al Grupo de Expertos de las Naciones Unidas en Nombres Geográficos.
} 
Pero lamentablemente, en los últimos años se observa cierto afán de 'recuperación' de formas arcaicas que ya habían quedado en desuso. Concretamente se aprovecha un foro colaborativo privado, Wikipedia, para dar nueva vida a grafías totalmente obsoletas (Vich, Guisona, Bañolas...) pretendiendo que se trata de topónimos castellanos. Un nombre de lugar catalán (o vasco o gallego), oficializado durante la época contemporánea y hasta el afianzamiento del Estado emanado de la Constitución de 1978, no se convierte por eso automáticamente en un nombre castellano. Una minoría de casos (al menos en Cataluña) sí fueron castellanizaciones evidentes, pero en general los nombres de lugar catalanes no se convirtieron por arte de magia en castellanos en el s. XIX y no lo son tampoco ahora. Es evidente la instrumentalización agresiva y anticatalana de esta maniobra (Burgueño y Aubet, 2017).

No podemos creer que para un castellanohablante sea más comprensible Vich que Vic o que le sea imposible pronunciar Vilanova en vez de Villanueva. La construcción de un espacio común de convivencia (algo que ciertamente a muchos no interesa en absoluto) debería comportar un mayor aprecio por las lenguas hispánicas no castellanas, y esto tiene una traslación muy clara en toponimia. Simplemente se trata de asumir la lógica de las recomendaciones de la RAE en el tratamiento de los topónimos ${ }^{74}$ entender que la mayoría de las formas antiguamente oficiales ni son propiamente castellanas (en nuestro caso generalmente son simples arcaísmos catalanes) ni merecen ser reivindicadas por los castellanohablantes, porque carecen de utilidad práctica. En cuanto al reducido número de burdas castellanizaciones inventadas a partir de 1720 , no merecen ningún aprecio, además de no contribuir en nada a la convivencia entre los pueblos del Estado. ${ }^{75}$

\section{Agradecimientos}

Proyecto de investigación de la Dirección General de Investigación Científica y Técnica, CSO2014-54078-C2-1-P.

74 http://www.rae.es/diccionario-panhispanico-de-dudas/que-contiene/tratamiento-de-los-toponimos

75 Aceptamos la contrapartida que propugna Vicenç Rosselló (2004: 33): «si volem que ens respecten, respectem els altres: parle, més que res, dels topònims castellans: si volem Girona i Lleida, no els freguem pels nassos Terol i Osca.» 


\section{BIBLIOGRAFÍA}

Basseda, L. (1990): Toponymie historique de Catalunya Nord, Prada, Universitat de Perpinyà-Terra Nostra, 796 pp.

Burgueño, J. (2008): El mapa com a llenguatge geogràfic. Recull de textos històrics (ss. XVII-XX), Barcelona, Societat Catalana de Geografia, CXXXIII+211 pp.

Burgueño, J. (2014): Nomenclátor que comprende las poblaciones... de España (1860). Edició de la informació referida a les terres de parla catalana, Barcelona, Societat Catalana de Geografia, 190 pp + CD.

Burgueño, J., Aubet, A. (2016): "Les reformes de la toponímia municipal impulsades per la Real Sociedad Geográfica (1916) i la Generalitat de Catalunya (1933). Crònica d'una errada ortogràfica: el Pinell... de Brai?", Treballs de la Societat Catalana de Geografia, 82, pp. 29-62.

Burgueño, J., Aubet, A. (2017): "Conflicte toponímic a Catalunya, encara ara. La reforma ortogràfica del català (1913) i els noms dels municipis”, Recerques, en prensa.

Caballero, F. (1834). Nomenclatura geogràfica de España. Anàlisis gramatical y filosófico de los nombres de pueblos y lugares de la Península, con aplicación a la topografía y a la historia, Madrid, Imp. Eusebio Aguado, 240 pp.

Coromines, J. (1989-97). Onomasticon cataloniae. Els noms de lloc i noms de persona de totes les terres de llengua catalana, Barcelona, Curial-La Caixa, 8 vol.

Galera i Monegal, M. (2006): "Estudi introductori a l'edició del facsímil del mapa Nova Principatus Cataloniae descriptio. El primer mapa imprès de Catalunya (16021605)", en Nova Principatus Cataloniae descriptio apud Ioannem Baptistam Vrints, Barcelona, ICC, 21 pp.

Gay Escoda, J. M. (1997): El corregidor a Catalunya, Madrid, Marcial Pons. $1.102 \mathrm{pp}$.

Generalitat de Catalunya (1933): Divisió territorial. Estudis i projectes. Nomenclàtor de municipis, Barcelona, Llibreria Bosch, 367 pp.

Iglésies, J. (1974): Estadístiques de població de Catalunya el primer vicenni del segle XVIII, Barcelona, Fundació Salvador Vives Casajuana, 3 vol.

Labèrnia, P. (1840): Diccionari de la llengua catalana ab la correspondencia castellana y llatina, Barcelona, Estampa dels hereus de la V. Pla, 2 vol.

Lladonosa, Josep (1977): Informe històric sobre els topònims Lérida-Lleida, Barcelona, Rafael Dalmau Editor, 62 pp.

Mancomunitat de Catalunya (1918): Nomenclàtor de les ciutats, viles i pobles de Catalunya, Barcelona, Imp. Casa de Caritat, 96 pp.

Madoz, P. (1845-50): Diccionario geográfico-estadístico-histórico de España y sus posesiones de Ultramar, Madrid, Madoz, 16 vol.

Mujica Ulazia, N. (2010): "Bilingüismo, toponimia y cartografía en la comunidad autónoma del País Vasco", en X. Sousa Fernández (ed.), Toponimia e cartografía, Santiago de Compostela, Consello da Cultura Galega, pp. 73-102. 
Nadal, F., Urteaga, L. (2012): "La toponímia", en Atles topogràfic-històric de Catalunya 1:50.000. Primeres edicions dels fulls de Catalunya del Mapa Topográfico de España, Barcelona, Institut Cartogràfic de Catalunya, pp. 47-50.

Pelissier, J.-P. (1986): Paroisses et communes de France. Pyrénées Orientales, París, CNRS.

Ruhstaller, S., Gordón Peral, M. D. (2013). "Transmisión y normalización de los nombres de lugar y su relevancia para la normalización toponímica" en M. D. Gordón Peral (ed.) Lengua, espacio y sociedad. Investigaciones sobre normalización toponímica en España, Berlín, De Gruyter, pp. 9-36.

Rosselló i Verger, V. M. (2004): Toponímia, geografia i cartografia, València, Universitat de València, 403 pp.

Rosselló i Verger, V. M. (2008). Cartografia històrica dels països catalans, València, Universitat de València-Institut d'Estudis Catalans, 402 pp.

[Santiago Palomares, F. J. de] (ed.) (1789): España dividida en provincias e intendencias, y subdividida en partidos, corregimientos, alcaldías mayores, gobiernos políticos y militares, así realengos como de órdenes, abadengo y señorío. Obra formada por las relaciones originales de los respectivos intendentes del Reyno, a quines se pidieron de orden de S. M. por el Exmo. Sr. conde de Floridablanca, y su ministerio de Estado en 22 de marzo de 1785, Madrid, Imp. Real, 2 vol.

Segarra, M. (1985a): Història de l'ortografia catalana, Barcelona, Empúries, 462 pp.

Segarra, M. (1985b): Història de la normativa catalana, Barcelona, Enciclopèdia Catalana, $222 \mathrm{pp}$.

Ullastra, J. (1980): Grammatica cathalána, Barcelona, Fundació Mediterrània, LXVIII+396 pp.

Fecha de recepción: 15 de julio de 2016.

Fecha de aceptación: 22 de marzo de 2017. 